\title{
Postoperative Endophthalmitis Caused by Staphylococcus haemolyticus following Femtosecond Cataract Surgery
}

\author{
Margaret Wong Benjamin R. Baumrind James H. Frank \\ Robert L. Halpern
}

Eye Consultants of Atlanta, Atlanta, Ga., USA

\section{Key Words}

Endophthalmitis · Staphylococcus haemolyticus - Femtosecond cataract surgery

\begin{abstract}
A 53-year-old Caucasian man underwent femtosecond cataract surgery and then presented with pain and hand motions vision 1 day following surgery. Anterior segment examination showed a 2-mm-layered hypopyon, a well-centered intraocular lens in the sulcus, and an obscured view to the fundus. B-scan ultrasonography showed significant vitritis and that the retina was attached. A tap and an injection of vancomycin $1 \mathrm{mg}$ per $0.1 \mathrm{ml}$ and of ceftazidime $2.25 \mathrm{mg}$ per $0.1 \mathrm{ml}$ were performed. The tap eventually yielded culture results positive for Staphylococcus haemolyticus, which was sensitive to vancomycin. We report a case of endophthalmitis that occurred on postoperative day 1 following complicated cataract surgery. This is an uncommon bacterium that is not widely reported in the literature as a cause of endophthalmitis in the postoperative period. We urge clinicians to consider S. haemolyticus as an offending agent, especially when the infection presents very early and aggressively in the postoperative period.

(C) 2015 The Author(s) Published by S. Karger AG, Basel
\end{abstract}

\section{Introduction}

Postoperative endophthalmitis following cataract surgery could have potentially devastating consequences of severe vision loss or even blindness. Endophthalmitis has an incidence rate between 0.1 and $0.2 \%$ [1-3]. In these cases, the most common pathogen is Staph-

KARGER 125/s $\begin{aligned} & \text { Margaret Wong, MD } \\ & \text { Eye Consultants of Atlanta } \\ & 3225 \text { Cumberland Boulevard, Suite } 800 \\ & \text { Atlanta, GA } 30339-3367 \text { (USA) } \\ & \text { E-Mail margaret.oph@gmail.com }\end{aligned}$


Wong et al.: Postoperative Endophthalmitis Caused by Staphylococcus haemolyticus following Femtosecond Cataract Surgery

ylococcus epidermidis $[4,5]$. The average time from surgery to presentation of endophthalmitis is usually 6-7 days [6,7]. We describe a case of immediate postoperative endophthalmitis from Staphylococcus haemolyticus. This is a rare cause of endophthalmitis in the postoperative setting following cataract surgery, with an incidence of $0.002 \%$ in the Endophthalmitis Vitrectomy Study [6]. Our case is unique in that this is the first case to our knowledge of $S$. haemolyticus endophthalmitis following femtosecond cataract surgery, and this rapidly progressive infection produced severe inflammation on postoperative day 1 .

\section{Case Report}

A 53-year-old Caucasian man presented for left eye blurry vision. His past ocular history was significant for right eye cataract surgery, right eye retinal tear and laser retinopexy, and bilateral high myopia. His past medical history was significant for renal cell carcinoma and nephrectomy. There were no medications and no drug allergies.

Best corrected visual acuity was $20 / 20$ and $20 / 40(-2)$, respectively. A right eye slit lamp examination was normal, while a left eye slit lamp examination showed 3+ nuclear sclerotic cataract. Intraocular pressures were 12 and $11 \mathrm{~mm} \mathrm{Hg}$, respectively. A right eye fundus examination showed peripheral laser scars at 10 o'clock, and a left eye fundus examination was normal. The patient was scheduled for left eye cataract surgery.

Cataract surgery was performed using the femtosecond laser to make the limbal relaxing incision, capsulotomy, and nucleus fragmentation. The eye was then prepped and draped in sterile ophthalmic fashion. Following hydrodissection, an anterior capsular tear was identified with extension to the posterior capsule. All nuclear fragments were removed. A complete anterior vitrectomy was performed, and there was no vitreous to the wounds. A sulcus intraocular lens was placed, and corneal wounds were sutured and well sealed.

On postoperative day 1 , vision was counting fingers at 2 feet. Intraocular pressure was $17 \mathrm{~mm} \mathrm{Hg}$. The anterior chamber had 4+ cells and 3+ fibrin anterior to the intraocular lens. A fundus examination was normal. The differential diagnoses included sterile, robust inflammatory response versus endophthalmitis. Observation was chosen at this time with the following medications: difluprednate 4 times a day, bromfenac once a day, cyclopentolate 4 times a day, and besifloxacin 6 times a day. Six hours later and still on postoperative day 1 , the patient experienced pain and decreased vision to hand motions. There was a 2-mm hypopyon and a completely obscured view of the fundus. B-scan ultrasonography showed 3+ vitritis and attached retina $360^{\circ}$. The working diagnosis was now endophthalmitis, and a tap and an injection of vancomycin $1 \mathrm{mg}$ per $0.1 \mathrm{ml}$ and of ceftazidime $2.25 \mathrm{mg}$ per $0.1 \mathrm{ml}$ were performed. A second tap and injection were performed on postoperative day 2 with the same antibiotics, since it was felt to be an aggressive organism that was progressing quickly. A Gram stain showed Gram-positive cocci in clusters. On postoperative day 3, the patient's vision was still hand motions, and there was no view of the fundus. However, the hypopyon reduced in size and a B-scan showed decreased vitritis. On postoperative day 7, culture results showed $S$. haemolyticus sensitive to vancomycin. A third injection of vancomycin was performed. The hypopyon and vitritis continued to improve daily, and vision improved to counting fingers.

On postoperative day 14, the patient complained of painless decreased vision in the left eye. Vision was hand motions, and the anterior chamber showed 1+ cells. A fundus examination showed 3+ vitritis and a complete retinal detachment. Pars plana vitrectomy and injection of $\mathrm{C}_{3} \mathrm{~F}_{8}$ gas was performed to repair the detachment. The patient continues to improve postoperatively. 
Wong et al.: Postoperative Endophthalmitis Caused by Staphylococcus haemolyticus following Femtosecond Cataract Surgery

\section{Discussion}

Endophthalmitis is a serious complication following cataract surgery that could result in devastating vision loss [8]. The average time from surgery to presentation of endophthalmitis is usually 6-7 days [6, 7]. The most common pathogen is S. epidermidis, but rarely other coagulase-negative bacteria are causative agents $[4,5]$. Coagulase-negative Staphylococcus is a normal skin and mucus membrane commensal, most commonly in the axillary, inguinal, and perineal regions. Possible virulence factors of coagulase-negative bacteria appear to be protease, lipase, slime production, and hemagglutination, and most cases of coagulasenegative Staphylococcus are sensitive to vancomycin [9].

S. haemolyticus is a Gram-positive, coagulase-negative bacterium that is uncommonly reported in the literature as a cause of endophthalmitis in the postoperative period. Schimel et al. [5] reported 448 cases of endophthalmitis, and less than $6 \%$ were caused by $S$. haemolyticus. In the Endophthalmitis Vitrectomy Study, 1 in 524 cases studied was caused by $S$. haemolyticus [6]. DeLima et al. [10] reported a case of endogenous $S$. haemolyticus endophthalmitis with no light perception vision that resulted in evisceration. Our case is unusual because there are very few cases of endophthalmitis caused by this bacterium, and there have been no cases of this bacterium causing endophthalmitis following femtosecond cataract surgery. In addition, the presentation of the endophthalmitis was on postoperative day 1 and progressed very quickly. Therefore, if a causative organism could be suspected early by its clinical presentation, the consequences may not be as severe, especially if treatment could be targeted even before culture results are obtained. It may also be beneficial to consider repeat intravitreal antibiotic injections of vancomycin, as was done in our case. We urge clinicians to consider $S$. haemolyticus as an offending agent, especially when the infection presents immediately and progresses aggressively in the postoperative period.

\section{Statement of Ethics}

The patient signed informed consent for the publication of this case report.

\section{Disclosure Statement}

The authors have no conflicts of interest to declare.

\section{References}

1 Kattan HM, Flynn HW, Pflugfelder SC, Robertson C, Forster RK: Nosocomial endophthalmitis survey. Ophthalmology 1991;98:227-238.

-2 Taban M, Behrens A, Newcomb RL, Nobe MY, Saedi G, Sweet PM, McDonnell PJ: Acute endophthalmitis following cataract surgery: a systematic review of the literature. Arch Ophthalmol 2005;123:613-620.

-3 West ES, Behrens A, McDonnell PJ, Tielsch JM, Schem OD: The incidence of endophthalmitis after cataract surgery among the US Medicare population increased between 1994 and 2001. Ophthalmology 2005;112:1388-1394.

4 Bannerman TL, Rhoden DL, McAllister SK, Miller JM, Wilson LA: The source of coagulase-negative Staphylococci in the Endophthalmitis Vitrectomy Study: a comparison of eyelid and intraocular isolates using pulsed-field gel electrophoresis. Arch Ophthalmol 1997;115:357-361.

5 Schimel AM, Miller D, Flynn HW: Endophthalmitis Isolates and antibiotic susceptibilities: a 10-year review of culture-proven cases. Am J Ophthalmol 2013;156:50-52. 
Wong et al.: Postoperative Endophthalmitis Caused by Staphylococcus haemolyticus following Femtosecond Cataract Surgery

6 Endophthalmitis Vitrectomy Study Group: Results of the Endophthalmitis Vitrectomy Study. A randomized trial of immediate vitrectomy and of intravenous antibiotics for the treatment of postoperative bacterial endophthalmitis. Arch Ophthalmol 1995;113:1479-1496.

7 Bode DD, Gelender H, Forster RK: A retrospective review of endophthalmitis due to coagulase-negative Staphylococci. Br J Ophthalmol 1985;69:915-919.

8 Heaven CJ, Mann PF, Boase DL: Endophthalmitis following extracapsular cataract surgery: a review of 32 cases. Br J Ophthalmol 1992;76:419-423.

-9 Ravindran P, Arumugam M, Yendremban RBS: Virulence, speciation and antibiotic susceptibility of ocular coagulase negative Staphylococci. J Clin Diagn Res 2014;8:33-37.

10 DeLima LM, Cecchetti SA, Cecchetti DF, Arroyo D, Romao EA, Dantos M, Neto MM: Endophthalmitis: a rare but devastating metastatic bacterial complication of hemodialysis catheter-related sepsis. Ren Fail 2012;34:119-122. 